\title{
INFLUÊNCIA DAS CARACTERÍSTICAS FÍSICO-QUÍMICAS E COMPOSIÇÃO ELEMENTAR NAS CORES DE MÉIS PRODUZIDOS POR Apis mellifera NO SUDOESTE DA BAHIA UTILIZANDO ANÁLISE MULTIVARIADA
}

\author{
Julian Júnio de Jesus Lacerda*, José Soares dos Santos, Shaiala Aquino dos Santos, Gisele Brito Rodrigues e Maria Lúcia \\ Pires dos Santos \\ Departamento de Ciências Naturais, Universidade Estadual do Sudoeste da Bahia, Estrada do Bem Querer, km 4, 45083-900 \\ Vitória da Conquista - BA, Brasil
}

Recebido em 29/4/09; aceito em 18/12/09; publicado na web em 8/4/10

\begin{abstract}
INFLUENCE OF PHYSICOCHEMICAL AND ELEMENTAL COMPOSITION ON HONEY COLORS PRODUCED BY Apis mellifera IN SOUTHWEST BAHIA USING MULTIVARIATE ANALYSIS. In this study honey samples produced in the southwest of Bahia were characterized based on physicochemical and mineral ( $\mathrm{Ca}, \mathrm{Mg}, \mathrm{Na}, \mathrm{K}, \mathrm{Mn}, \mathrm{Fe}$ and $\mathrm{Zn}$ ) composition. The metals were determined by atomic absorption spectrophotometry. The application of multivariate analysis showed that the honey colors are consequence of the mineral and physicochemical compositions. The darkest honey samples are characterized by higher values of $\mathrm{pH}$ and for presenting a strong relationship with $\mathrm{Ca}$ and $\mathrm{Fe}$ content.
\end{abstract}

Keywords: honey; physicochemical composition; principal component analysis.

\section{INTRODUÇÃO}

O mel é um produto alimentício composto por uma solução concentrada de açúcares com predominância de glicose e frutose. Contém ainda uma mistura complexa de outros carboidratos, enzimas, aminoácidos, ácidos orgânicos, minerais, substâncias aromáticas, pigmentos e grãos de pólen, podendo conter cera de abelhas procedente do processo de extração. ${ }^{1}$

$\mathrm{Na}$ aquisição do mel um dos principais critérios adotados pelos consumidores é a aparência, sendo que o produto de cor clara alcança valor mais elevado no mercado mundial. No entanto, os méis de cor escura são mais ricos em sais minerais, ${ }^{2}$ em vitaminas $\mathrm{B}$ e C, ${ }^{3} \mathrm{e}$ possuem um aroma mais acentuado que os méis de cor clara. A cor é uma propriedade óptica, resultante da absorção de luz nos diferentes comprimentos de onda provocados pelos constituintes do mel. ${ }^{4}$ Este parâmetro está relacionado com a origem floral, variações climáticas durante o fluxo do néctar e amadurecimento do mel na colméia, ${ }^{5}$ podendo variar de quase incolor a pardo-escura, conforme a instrução normativa $\mathrm{n}^{\circ} 11$ de 20/10/2000. ${ }^{6}$

Para alguns autores a cor original do mel com relação ao seu valor natural varia muito e não é fator de seleção da qualidade. ${ }^{7}$ Isso porque o envelhecimento é um fator que acentua a cor do mel. ${ }^{8}$ Porém, Azeredo et al. estudaram as características físico-químicas de méis em São Fidelis, estado do Rio de Janeiro, e demonstram não haver nenhum tipo de variação com relação à cor, tanto na absorbância quanto na observação visual das amostras em análises realizadas em intervalos regulares de 90 dias por 365 dias. ${ }^{9}$ Moura et al. estudaram as alterações sofridas por méis armazenados em temperatura ambiente em Teresina, Piauí $\left(30-42{ }^{\circ} \mathrm{C}\right)$ durante 6 meses, analisando-os de 3 em 3 meses no que se refere às variações na faixa de cor e mostraram que no armazenamento nessas condições a cor dos méis permaneceu na mesma classificação, embora a absorbância tenha aumentado $21,5 \% \cdot{ }^{10} \mathrm{~A}$ intensificação da coloração do mel é um reflexo das reações de substâncias polifenólicas com sais de ferro, do conteúdo de minerais e da instabilidade da frutose em solução ácida, sendo a cor mais escura um indicador da presença de alto conteúdo de minerais. ${ }^{11}$

\footnotetext{
*e-mail: julianlacerda@gmail.com
}

A região sudoeste da Bahia apresenta uma flora bastante diversificada, onde se pode obter méis e outros produtos apícolas em quantidades e características diferentes. A apicultura vem se desenvolvendo como uma atividade econômica geradora de emprego e renda para famílias de pequenos proprietários rurais ao complementar as outras ocupações agropecuárias, principalmente na época da seca. Além disso, tem baixo impacto ambiental, o que permite a utilização permanente dos recursos naturais. O principal produto apícola produzido na região é o mel, sendo reduzido o aproveitamento dos outros produtos apícolas diretos (geleia real, pólen, própolis, apitoxina e cera) que diversificariam e poderiam agregar valor à cadeia produtiva.

Este estudo pode contribuir na identificação e melhoria do padrão de qualidade dos méis produzidos nessa região, fornecendo informações para a sua padronização e valorização. Assim, o objetivo deste trabalho foi correlacionar a cor dos méis com as outras características físico-químicas, aplicando análise multivariada em amostras provenientes da região sudoeste da Bahia.

\section{PARTE EXPERIMENTAL}

\section{Origem das amostras}

Foram coletadas 24 amostras de méis de abelhas Apis mellifera de origem monofloral e polifloral, adquiridas em contato direto com apicultores nos meses de março a agosto de 2007. Cada frasco contendo $250 \mathrm{~g}$ de mel foi identificado com a data da produção, local de coleta e florada predominante. Os méis foram produzidos em 8 municípios da região sudoeste do Estado da Bahia: 5 amostras em Barra do Choça, florada de Aroeira; 4 em Jequié, florada de Unha de gato; 1 em Anagé, florada de Vassourinha; 10 em Vitória da Conquista, 1 em Mortugaba, 1 em Ribeirão do Largo, 1 em Piripá e 1 em Belo Campo, floradas predominantemente silvestres.

A Figura 1S, material suplementar, mostra o mapa dos locais de coleta das amostras estudadas. A região se caracteriza por uma economia predominantemente agrícola e pela existência de uma diversidade de espécies botânicas, devido à transição climática da Zona de Mata Atlântica para o semiárido baiano.

As amostras foram armazenadas em frascos de polietileno e mantidas em temperatura ambiente (média de $23^{\circ} \mathrm{C}$ ), por no máximo 
30 dias, até a determinação de cor, umidade, sólidos solúveis, cinzas, CE e acidez livre, já que o tempo de estocagem, a luz, o calor e as possíveis reações enzimáticas poderiam afetar a cor dos méis. Para a determinação dos elementos químicos as amostras ficaram armazenadas por até 3 meses nas mesmas condições.

\section{Reagentes e soluções}

Para a determinação da acidez livre nas amostras de méis foi utilizado hidróxido de sódio ( $\mathrm{NaOH}, 97 \%, \mathrm{~m} / \mathrm{m}$, Vetec, Rio de Janeiro, RJ, Brasil). O procedimento de extração foi realizado com ácido nítrico $\left(\mathrm{HNO}_{3}, 65 \%\right.$, m/v, P.A., Merck, Rio de Janeiro, RJ, Brasil) e peróxido de hidrogênio $\left(\mathrm{H}_{2} \mathrm{O}_{2}, 29 \%\right.$ m/v, P.A., Synth, Diadema, SP, Brasil).

Para a determinação de metais, foram preparadas soluções estoque de concentração $100 \mathrm{mg} \mathrm{L}^{-1}$ a partir de soluções padrão (Accustandart/SpecSol) de concentração $1000 \mathrm{mg} \mathrm{L}^{-1}$. As soluções de trabalho foram obtidas por diluição serial das soluções estoque e usadas como soluções de referência para obtenção das curvas analíticas. O meio de preparação das soluções para calibração foi ácido nítrico $1 \%(\mathrm{v} / \mathrm{v})$. Todas as soluções foram preparadas utilizando água deionizada purificada através do sistema Milli-Q (Millipore) até alta pureza (resistividade $18 \mathrm{M} \Omega \mathrm{cm}^{-1}$ ).

\section{Preparo das amostras}

Toda a vidraria e os frascos de polietileno foram lavados com soluções de detergente, e foram imersos por $24 \mathrm{~h}$ em uma solução de ácido nítrico $10 \%$ (v/v). Logo após, o material foi enxaguado com água deionizada e seco ao ar livre. A extração dos minerais contidos nas amostras de méis foi realizada sob via úmida com agitação ultrassônica, conforme Santos et al., ${ }^{12}$ que demonstraram não haver diferença significativa entre a digestão das amostras de méis sob via úmida e sob via seca. Pesou-se 1,25 g de mel em uma balança analítica Micronal, modelo PB303, adicionou-se 1,25 mL de $\mathrm{HNO}_{3}$ e 2,5 $\mathrm{mL}$ de $\mathrm{H}_{2} \mathrm{O}_{2}$. As amostras foram sonicadas por $20 \mathrm{~min}$ em um banho ultrassônico (frequência $40 \mathrm{kHz}, 320 \mathrm{~W}$, USC-1800, Unique, São Paulo, Brasil). O extrato foi transferido para um balão volumétrico de $25 \mathrm{~mL}$ e diluído, com água deionizada até o menisco. Para cada amostra foi realizada extração em triplicata.

\section{Procedimentos analíticos}

A determinação das concentrações dos elementos $\mathrm{Ca}, \mathrm{Mg}, \mathrm{Na}$, $\mathrm{K}, \mathrm{Mn}, \mathrm{Fe}$ e $\mathrm{Zn}$ foi realizada diretamente no extrato das amostras empregando um espectrômetro de absorção atômica por chama (F AAS) Perkin Elmer, modelo AAnalyst 200 (USA) equipado com corretor de fundo de deutério. O sistema de aquisição de dados foi gerenciado por microcomputador acoplado ao F AAS. A fonte de radiação empregada foi uma lâmpada de cátodo oco. Com base nas recomendações do fabricante, a chama ar-acetileno foi utilizada na determinação dos 7 elementos em estudo. Para obtenção dos sinais de absorção do K, Ca, Mg e Zn foram utilizados os seguintes parâmetros: largura de fenda de $0,7 \mathrm{~nm}$ e comprimentos de onda 766, 423, 285 e $214 \mathrm{~nm}$, respectivamente. Para $\mathrm{Mn}$ e Fe, a largura da fenda foi de 0,2 nm e os comprimentos de onda de 280 e $401 \mathrm{~nm}$, respectivamente. Finalmente, para $\mathrm{Na}$, o comprimento de onda utilizado foi de 589 $\mathrm{nm}$ e abertura de fenda de $0,2 \mathrm{~nm}$. Para controlar a ionização que ocorre durante a determinação de $\mathrm{Na}$ e K por F AAS, adicionou-se nos padrões e nas amostras, $\mathrm{CsCl}(0,1 \% \mathrm{~m} / \mathrm{v})$. Para evitar a interferência de alguns elementos existentes na matriz durante a determinação de $\mathrm{Ca}$ e $\mathrm{Mg}$, adicionou-se $\mathrm{LaCl}_{2}(0,1 \% \mathrm{~m} / \mathrm{v})$ nos padrões e nas amostras.

O F AAS foi calibrado com curvas analíticas usando soluções preparadas com intervalos de concentração de 0,5 a $1,0 \mathrm{mg} \mathrm{L}^{-1}$ para os elementos $\mathrm{Na}, \mathrm{K}$, Fe e $\mathrm{Mn}$, para $\mathrm{Mg}$ de 0,25 a 2,0 $\mathrm{mg} \mathrm{L}^{-1}$, para $\mathrm{Zn}$ de 0,5 a $5,0 \mathrm{mg} \mathrm{L}^{-1}$ e para Ca de 1,0 a $20,0 \mathrm{mg} \mathrm{L}^{-1}$.

Para verificação da cor do mel utilizou-se o método de Bianchi, ${ }^{13}$ que consiste na medida da absorbância a $635 \mathrm{~nm}$ de uma solução $50 \%(\mathrm{~m} / \mathrm{v})$ de mel em água. Após a diluição, a solução foi deixada em repouso por $15 \mathrm{~min}$ antes da determinação da absorbância. O espectrofotômetro UV-VIS, modelo E-225D, foi calibrado com água ultrapura e a classificação foi dada pela escala de Pfund. A condutividade elétrica foi determinada segundo as recomendações da AOAC, ${ }^{14}$ para isso se utilizou um condutivímetro de bancada CAAL, modelo MCA-150. Os teores de cinzas foram determinados pela relação linear existente entre a condutividade elétrica e as cinzas, conforme a Equação 1:15

$$
\mathrm{C}=0,14+1,74 \mathrm{~A}
$$

onde $\mathrm{C}$ é a condutividade elétrica em miliSiemens $\mathrm{cm}^{-1} \mathrm{e} \mathrm{A}$ é o conteúdo de cinzas em g/100 g de mel.

A umidade e a acidez livre foram determinadas, segundo métodos do item $n^{\circ} 969.38$ b da AOAC. ${ }^{14}$ As determinações dos sólidos solúveis totais ( $\left.{ }^{\circ} \mathrm{Brix}\right)$ e dos teores de umidade foram realizadas, utilizandose de um refratômetro ABBE de bancada Quimis, modelo: Q 767B, após a homogeneização da amostra. Os valores de $\mathrm{pH}$ foram obtidos em um pHmetro de bancada Digimed, modelo DM 20, conforme metodologia estabelecida pela International Honey Commission. ${ }^{16}$ Todas as análises foram determinadas em triplicata e os valores foram usados para a avaliação estatística.

\section{Avaliação estatística dos dados}

Devido à existência de diferentes fatores que influenciam na composição da cor do mel, as análises de correlação (Pearson r), componentes principais (ACP) e agrupamento hierárquico (AAH) foram aplicadas nos resultados das amostras analisadas, visando desenvolver um estudo de caracterização das variáveis indicativas da coloração dos méis em estudo.

Para interpretação dos dados, utilizando ACP e AAH, foi gerada uma matriz de dados 24x13 onde as linhas correspondem às amostras de méis (casos) e as colunas correspondem às variáveis (cinzas, $\mathrm{CE}$, umidade, sólidos solúveis, $\mathrm{pH}$, acidez, $\mathrm{Ca}, \mathrm{Mg}, \mathrm{Na}, \mathrm{K}, \mathrm{Fe}, \mathrm{Mn}$ e $\mathrm{Zn}$ ). Como as variáveis utilizadas não possuem a mesma escala, foi necessário escalonar os dados através do método Z-Score, conforme a Equação 2:

$$
\mathrm{Z}=\frac{\mathrm{X}-\mathrm{X}_{\mathrm{m}}}{\sigma}
$$

onde, $Z$ é o valor padronizado da variável, $X$ é o valor inicialmente encontrado da variável para cada amostra, $X_{m}$ é o valor médio da variável e $\sigma$ é o desvio padrão da variável na amostra. Na AAH foi utilizada a técnica de análise de cluster que se baseia na partição de uma população heterogênea em vários grupos homogêneos. No agrupamento não há classes pré-definidas, os elementos são agrupados de acordo com a semelhança. A medida de similaridade usada foi a distância Euclidiana quadrática e método hierárquico aglomerativo usado foi o método de ligação de Ward.

A análise em componentes principais foi utilizada para obter uma transformação mais representativa e compacta das observações. Os componentes são individualmente responsáveis pela variância dos dados. Geralmente a maior parte da variância pode ser explicada por um número reduzido de componentes, sendo possível o descarte do restante sem perda significativa de informação. A partir da ACP puderam ser extraídos os autovalores e os autovetores da matriz de covariância do grupo de variáveis originais. 
Os componentes principais (PCs) são as variáveis descorrelacionadas, obtidas multiplicando-se as variáveis correlacionadas originalmente com os autovetores (loadings). Os autovalores dos PCs são as medidas das discrepâncias associadas à participação das variáveis originais nos PCs. Os elementos de transformação das novas variáveis são chamados de escores. ${ }^{17}$

A análise de correlação (Pearson $r$ ) foi realizada entre as variáveis cor, cinzas, $\mathrm{CE}$, umidade, sólidos solúveis, $\mathrm{pH}$, acidez, $\mathrm{Ca}, \mathrm{Mg}, \mathrm{Na}$, $\mathrm{K}, \mathrm{Fe}, \mathrm{Mn}$ e Zn.

Todas as operações matemáticas e estatísticas foram realizadas com auxílio dos programas SPSS 15.0 (Statistical Package for Social Sciences) e Excel 2007.

\section{RESULTADOS E DISCUSSÃO}

As amostras estudadas representaram todas as classes de cores de méis estabelecidas pela classificação de Pfund, conforme a Figura 1, o que demonstra a diversidade florística desta região, que se caracteriza como região de transição climática entre a zona de mata atlântica e o semiárido baiano. Houve predominância das cores âmbar-escuro e branco, 29 e $25 \%$, respectivamente. Isto está relacionado à época do ano - nos meses de maio e junho ocorre a florada da aroeira com produção de méis de tonalidade mais escura, nos meses de novembro a março ocorrem floradas de unha de gato, com a produção de méis de cor clara. A florada silvestre também ocorre nos meses de novembro a março, mas origina méis de diferentes tonalidades, sendo que o mel de cor clara é comercializado por um valor de mercado maior que o mel escuro. As classes de cores encontradas estão em conformidade com a legislação, que considera aceitáveis variações de branco-água a âmbar-escuro. ${ }^{6}$

A Tabela 1 apresenta os valores médios das características físicoquímicas cor, cinzas, $\mathrm{CE}$, umidade, sólidos solúveis, $\mathrm{pH}$, acidez, $\mathrm{Ca}, \mathrm{Mg}, \mathrm{Na}, \mathrm{K}, \mathrm{Fe}, \mathrm{Mn}$ e $\mathrm{Zn}$ nas amostras estudadas. Os teores de

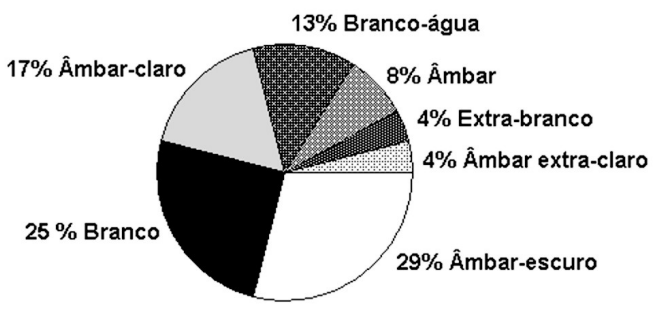

Figura 1. Distribuição percentual das classes de cores obtidas nas amostras de méis

cinzas encontrados nas amostras estudadas variaram entre $0,09 \mathrm{e}$ $0,64 \%(\mathrm{~m} / \mathrm{m})$ sendo que apenas uma amostra apresentou valor acima de $0,60 \%(\mathrm{~m} / \mathrm{m})$, limite estabelecido pela legislação brasileira. ${ }^{6}$ Os valores de condutividade elétrica $\left(175,40\right.$ a $\left.1170,00 \mu \mathrm{S} \mathrm{cm}^{-1}\right)$ são semelhantes aos encontrados por outros pesquisadores em amostras de méis da região do litoral norte do estado da Bahia (271,67 a 1634,00 $\left.\mu \mathrm{S} \mathrm{cm} \mathrm{cm}^{-1}\right) .{ }^{18}$ As porcentagens de umidade variaram de 17,00 a $21,2 \%$ $(\mathrm{m} / \mathrm{m})$, estando a maioria das amostras $(79 \%)$ abaixo do limite máximo de $20,0 \%(\mathrm{~m} / \mathrm{m})$ estabelecido pela legislação brasileira, indicando que as mesmas se encontram preservadas. ${ }^{6} \mathrm{O}$ teor de umidade do mel influencia positivamente sobre o desenvolvimento de leveduras contribuindo, consequentemente, para a fermentação do produto, determinando também seu prazo de validade. ${ }^{8,15}$ Os teores de sólidos solúveis totais variaram de 77,00 a $81,30 \%(\mathrm{~m} / \mathrm{m})$. As medidas de $\mathrm{pH}$ encontradas nas amostras variaram de 3,64 a 5,3, valores semelhantes aos obtidos por Mendonça, em diferentes méis do estado de São Paulo. ${ }^{19}$ Os valores de acidez encontrados nas amostras variaram de 15,65 a 61,00 meq kg-1, a maioria das amostras (84\%) apresentou acidez dentro do limite estabelecido pela legislação que é de 50,0 meq $\mathrm{kg}^{-1}$, parâmetro que indica também o nível de preservação do mel.

Tabela 1. Características físico-químicas de amostras de méis de Apis mellifera provenientes da região Sudoeste da Bahia

\begin{tabular}{|c|c|c|c|c|c|c|c|c|c|c|c|c|c|c|}
\hline Amostra & $\mathrm{pH}$ & Cor & Umidade & SS & Acidez & $\mathrm{CE}$ & Cinza & $\mathrm{Ca}$ & $\mathrm{Mg}$ & $\mathrm{Na}$ & K & $\mathrm{Fe}$ & $\mathrm{Zn}$ & $\mathrm{Mn}$ \\
\hline & ---- & (mmPfund) & $(\%)$ & $\left({ }^{0}\right.$ Brix $)$ & $\left(\mathrm{Meq} \mathrm{Kg}^{-1}\right)$ & $\left(\mu \mathrm{S} \mathrm{m}^{-1}\right)$ & $(\%)$ & $\left(\mathrm{mg} \mathrm{Kg}^{-1}\right)$ & $\left(\mathrm{mg} \mathrm{Kg}^{-1}\right)$ & $\left(\mathrm{mg} \mathrm{Kg}^{-1}\right)$ & $\left(\mathrm{mg} \mathrm{Kg}^{-1}\right)$ & $\left(\mathrm{mg} \mathrm{Kg}^{-1}\right)$ & $\left(\mathrm{mg} \mathrm{Kg}^{-1}\right)$ & $\left(\mathrm{mg} \mathrm{Kg}^{-1}\right)$ \\
\hline 1 & 4,08 & 22,95 & 17,7 & 80,5 & 31,65 & 522,1 & 0,26 & 46,5 & 36,9 & 318,8 & 1575,6 & 3,8 & 13,5 & 7,8 \\
\hline 2 & 3,73 & 19,61 & 18,8 & 79,5 & 46,50 & 464,1 & 0,24 & 46,6 & 14,3 & 238,0 & 1506,4 & 5,4 & 57,6 & 9,4 \\
\hline 3 & 3,91 & 128,43 & 19,2 & 79,0 & 54,65 & 619,9 & 0,33 & 101,5 & 128,4 & 284,4 & 1427,2 & 4,4 & 55,3 & 10,7 \\
\hline 4 & 4,81 & 138,45 & 17,8 & 80,2 & 18,65 & 612,6 & 0,33 & 92,6 & 303,0 & 160,0 & 1270,4 & 10,4 & 62,5 & 11,0 \\
\hline 5 & 3,68 & 78,29 & 19,4 & 78,6 & 61,00 & 581,2 & 0,31 & 72,0 & 38,7 & 340,0 & 806,0 & 3,5 & 49,2 & 13,0 \\
\hline 6 & 4,26 & 47,46 & 19,8 & 78,2 & 49,15 & 1170,0 & 0,63 & 103,7 & 98,8 & 480,4 & 1348,0 & 3,6 & 62,2 & 13,0 \\
\hline 7 & 4,00 & 57,49 & 19,8 & 78,2 & 57,50 & 979,5 & 0,53 & 65,4 & 77,8 & 388,0 & 1430,4 & 6,6 & 55,3 & 16,6 \\
\hline 8 & 3,73 & $<0,10$ & 18,2 & 80,0 & 19,65 & 175,4 & 0,09 & 25,7 & 11,0 & 2650,0 & 4058,8 & 3,6 & 50,7 & 14,6 \\
\hline 9 & 3,79 & 110,6 & 21,2 & 77,0 & 42,15 & 494,7 & 0,26 & 59,1 & 23,6 & 224,4 & 1321,6 & 11,0 & 80,4 & 18,0 \\
\hline 10 & 3,79 & 79,03 & 20,4 & 77,8 & 52,75 & 594,0 & 0,32 & 33,6 & 17,8 & 262,8 & 1444,0 & 3,8 & 88,6 & 20,3 \\
\hline 11 & 3,93 & 106,51 & 20,8 & 77,5 & 28,00 & 439,1 & 0,23 & 17,4 & 32,8 & 183,6 & 1143,2 & 10,7 & 63,7 & 20,3 \\
\hline 12 & 3,74 & $<0,10$ & 17,8 & 80,4 & 18,65 & 193,6 & 0,10 & 16,2 & 11,6 & 2596,4 & 3652,4 & 3,0 & 43,6 & 20,1 \\
\hline 13 & 3,75 & $<0,10$ & 18,0 & 80,2 & 19,30 & 179,4 & 0,09 & 17,6 & 10,8 & 2634,0 & 3319,6 & 2,7 & 44,0 & 19,2 \\
\hline 14 & 4,48 & 79,40 & 17,8 & 80,4 & 22,80 & 563,3 & 0,30 & 58,3 & 27,9 & 184,0 & 1400,8 & 4,4 & 39,0 & 20,9 \\
\hline 15 & 4,86 & 161,85 & 17,4 & 80,6 & 21,25 & 701,1 & 0,38 & 132,3 & 37,9 & 198,4 & 1536,0 & 7,7 & 39,8 & 22,4 \\
\hline 16 & 3,64 & 12,18 & 20,8 & 77,5 & 42,50 & 342,0 & 0,19 & 39,7 & 15,4 & 184,8 & 894,8 & 1,7 & 40,9 & 18,6 \\
\hline 17 & 4,59 & 127,31 & 17,4 & 81,0 & 22,65 & 631,2 & 0,35 & 112,7 & 32,2 & 190,4 & 1303,2 & 6,2 & 9,4 & 20,3 \\
\hline 18 & 3,83 & 24,44 & 19,4 & 79,0 & 26,50 & 365,6 & 0,20 & 41,2 & 27,9 & 131,2 & 902,0 & 1,2 & 57,2 & 19,1 \\
\hline 19 & 4,80 & 147,37 & 17,2 & 81,0 & 20,50 & 587,7 & 0,31 & 111,9 & 32,8 & 162,0 & 1134,0 & 7,4 & 10,7 & 19,8 \\
\hline 20 & 5,30 & 167,79 & 17,5 & 80,8 & 15,65 & 666,5 & 0,37 & 129,5 & 39,6 & 178,4 & 1318,8 & 7,1 & 4,7 & 22,5 \\
\hline 21 & 3,70 & 22,95 & 20,4 & 79,0 & 46,25 & 336,2 & 0,18 & 51,9 & 15,5 & 167,6 & 864,8 & 0,9 & 6,5 & 18,7 \\
\hline 22 & 4,90 & 162,59 & 17,0 & 81,3 & 22,00 & 721,6 & 0,39 & 131,9 & 37,3 & 197,6 & 1506,4 & 4,5 & 9,7 & 20,7 \\
\hline 23 & 4,06 & 32,24 & 18,2 & 80,0 & 20,00 & 282,2 & 0,15 & 34,3 & 21,5 & 186,0 & 849,6 & $<0,1$ & $<0,02$ & 19,5 \\
\hline 24 & 4,10 & 31,12 & 17,4 & 81,0 & 16,30 & 276,9 & 0,14 & 31,7 & 21,3 & 130,0 & 811,2 & $<0,1$ & $<0,02$ & 17,9 \\
\hline
\end{tabular}


$\mathrm{Na}$ análise do conteúdo mineral do mel o potássio foi o mineral de concentração mais elevada nas amostras estudadas, com um valor médio de $1534,38 \mathrm{mg} \mathrm{kg}^{-1}$, seguido pelo sódio $527,96 \mathrm{mg} \mathrm{kg}^{-1}$, cálcio $65,96 \mathrm{mg} \mathrm{kg}^{-1}$, magnésio $46,46 \mathrm{mg} \mathrm{kg}^{-1}$ e ferro $4,73 \mathrm{mg} \mathrm{kg}^{-1}$. Essas concentrações são similares às encontradas em méis espanhóis avaliados por Gonzaalez-Miret et al. ${ }^{20}$

\section{Correlação entre as cores dos méis e os outros parâmetros físico-químicos}

A Tabela 2 mostra a matriz de correlação entre a cor e as variáveis umidade, $\mathrm{pH}$, sólidos solúveis, acidez livre, cinzas, $\mathrm{CE}, \mathrm{Ca}, \mathrm{Mg}, \mathrm{Na}$, $\mathrm{K}$, Fe e Zn. Observa-se que os parâmetros que apresentaram correlações mais significativas com a cor foram $\mathrm{Ca}(\mathrm{r}=0,822), \mathrm{pH}(\mathrm{r}=0,783)$, $\mathrm{Fe}(\mathrm{r}=0,671)$, cinzas $(\mathrm{r}=0,525), \mathrm{CE}(\mathrm{r}=0,524)$ e $\mathrm{Na}(\mathrm{r}=-0,500)$. Nas amostras avaliadas, a correlação positiva entre a cor e os teores de cinzas indica que a coloração escura dos méis está relacionada com os teores de minerais, conforme trabalhos apresentados na literatura. ${ }^{21}$ No entanto, devido à elevada correlação apresentada entre os pares cor-Ca e cor-Fe, ficou evidente que entre os minerais avaliados, $\mathrm{Ca}$ e Fe são os elementos que mais contribuem na intensidade das cores dos méis estudados. Assim, a cor escura do mel é um reflexo do teor de cinzas e, mais especificamente, das concentrações de Ca e Fe. Já a correlação negativa apresentada entre a cor e a concentração do $\mathrm{Na}$ indica que os méis claros também podem apresentar elevados teores de cinzas, compostas principalmente por $\mathrm{K}$ e $\mathrm{Na}$. Isso mostra que as cores dos méis estão relacionadas aos elementos contidos em sua matriz e não apenas à quantidade total de resíduos minerais.

Tabela 2. Matriz de correlação das variáveis, para as amostras de méis estudadas

\begin{tabular}{|c|c|c|c|c|c|c|c|}
\hline & Cor & Umid & Brix & $\mathrm{pH}$ & Acidez & $\mathrm{CE}$ & Cinzas \\
\hline Cor & 1 & & & & & & \\
\hline Umid & $-0,249$ & 1 & & & & & \\
\hline Brix & 0,207 & $-0,978 * *$ & 1 & & & & \\
\hline $\mathrm{pH}$ & $0,783 * *$ & $-0,643^{* *} *$ & $=0,613^{* *}$ & 1 & & & \\
\hline Acidez & $-0,131$ & $0,712^{* *}$ & $-0,725^{* * *}$ & $-0,516^{* *}$ & 1 & & \\
\hline $\mathrm{CE}$ & $0,524 * *$ & 0,057 & $-0,137$ & $0,469 *$ & $0,405^{*}$ & 1 & \\
\hline \multirow[t]{2}{*}{ Cinzas } & $0,525^{* *}$ & 0,051 & $-0,129$ & $0,478 *$ & 0,395 & $0,999 * *$ & 1 \\
\hline & Cor & $\mathrm{Ca}$ & $\mathrm{Mg}$ & $\mathrm{Na}$ & K & $\mathrm{Fe}$ & $\mathrm{Zn}$ \\
\hline Cor & 1 & & & & & & \\
\hline $\mathrm{Ca}$ & $0,822 * *$ & 1 & & & & & \\
\hline $\mathrm{Mg}$ & 0,367 & 0,357 & 1 & & & & \\
\hline $\mathrm{Na}$ & $-0,500 *$ & $-0,441^{*}$ & $-0,208$ & 1 & & & \\
\hline $\mathrm{K}$ & $-0,358$ & $-0,318$ & $-0,160$ & $0,956^{* * *}$ & 1 & & \\
\hline $\mathrm{Fe}$ & $0,671^{* *} *$ & 0,383 & $0,407 *$ & $-0,199$ & $-0,046$ & 1 & \\
\hline $\mathrm{Zn}$ & $-0,074$ & $-0,254$ & 0,245 & 0,137 & 0,176 & 0,375 & 1 \\
\hline
\end{tabular}

*Significativo ao nível de $5 \%$ probabilidade, pelo teste t de Student.

**Significativo ao nível de $1 \%$ probabilidade, pelo teste $\mathrm{t}$ de Student.

\section{Análise de agrupamento}

A análise de cluster foi utilizada para explorar a similaridade entre as amostras, definindo-as em grupos, considerando simultaneamente todas as variáveis. Preliminarmente, os valores das variáveis $\mathrm{Na}, \mathrm{K}, \mathrm{Ca}, \mathrm{Mg}, \mathrm{Fe}, \mathrm{Zn}, \mathrm{Mn}$, pH, acidez livre, cinzas, CE, umidade e sólidos solúveis foram normalizados através da transformação Zescores, com o objetivo de se atribuir um mesmo peso a cada uma delas. Na matriz inicial, os coeficientes de similaridade representam o grau de semelhança entre os pares de amostras e as mesmas foram arranjadas de acordo com os respectivos graus de similaridade, de modo que ficassem agrupadas segundo uma disposição hierárquica.
Os resultados foram organizados em gráfico do tipo dendrograma, mostrando as relações entre as amostras agrupadas.

O dendrograma da Figura 2 mostra três agrupamentos distintos de amostras de méis. O grupo I é formado pelas amostras de méis com a coloração mais clara (branco-água) de acordo com a escala de Pfund. O grupo II é formado por amostras de méis nitidamente mais escuros, classificam-se como âmbar-escuro. O grupo III é formado por amostras de coloração intermediárias da escala de Pfund, reunindo amostras de coloração âmbar extra-claro e âmbar-claro, branco, âmbar-claro e âmbar. O dendrograma é uma projeção simplificada em duas dimensões de uma relação n-dimensional, por isso é inevitável que apareçam algumas distorções quanto à similaridade. Esta análise evidencia que a cor dos méis está correlacionada com os outros parâmetros físico-químicos, sendo um indicador da composição do produto.

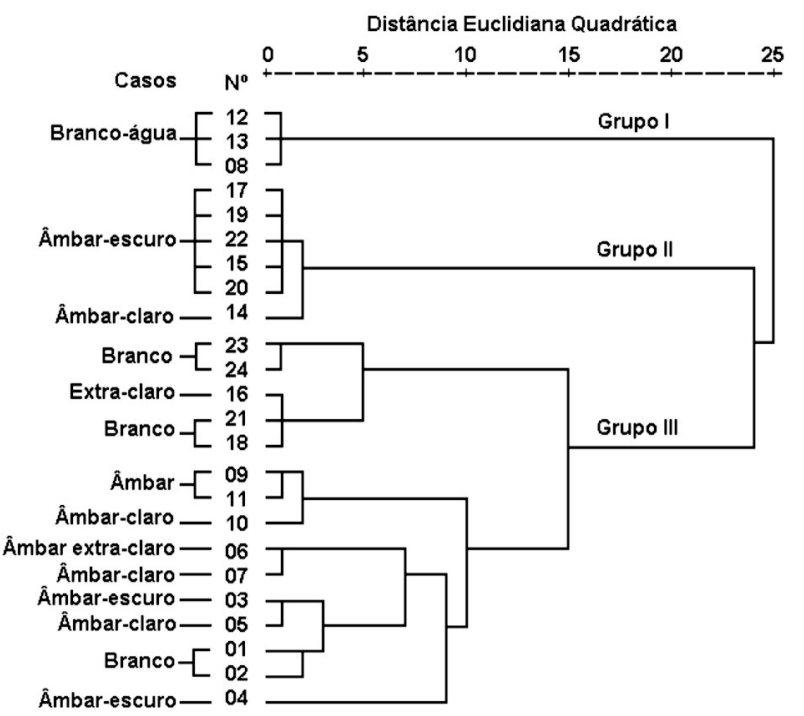

Figura 2. Dendrograma de análise de cluster entre variáveis, agrupando pela coloração as amostras dos méis estudados

\section{Análise de componentes principais}

Para complementar a classificação obtida através da análise de agrupamento, a matriz de dados com as variáveis $\mathrm{Na}, \mathrm{K}, \mathrm{Ca}, \mathrm{Mg}$, $\mathrm{Fe}, \mathrm{Zn}, \mathrm{Mn}, \mathrm{pH}$, acidez, cinzas, CE, umidade e sólidos solúveis foi escalonada por Z-escores e processou-se a análise de componentes principais (ACP). Foram selecionadas três componentes derivadas por rotação varimax, onde o primeiro componente concentrou $32,37 \%$ da variância total, o segundo $30,21 \%$ e o terceiro $13,58 \%$, acumulando $76,16 \%$ na variância total.

A distribuição das amostras de méis pela coloração em relação às componentes I, II e III é mostrada na Figura 3. Observa-se claramente a formação de três grupos bem definidos: o primeiro, formado unicamente pelas amostras de méis de coloração escura; o segundo, formado por amostras de coloração âmbar e o terceiro grupo formado por amostras de méis com coloração clara. Assim, ficou evidente que as variações das características físico-químicas e do conteúdo de minerais influenciam na separação dos agrupamentos ao longo das componentes 1 e 2, conforme a Figura 3.

O gráfico de loadings (Figura 4) permite a caracterização de tendências entre as variáveis. Observa-se ao longo do eixo da CP1 que as variáveis $\mathrm{Ca}, \mathrm{Fe}$, cinzas e $\mathrm{CE}$ influenciam diretamente os valores positivos que são as variáveis que se correlacionam com as amostras de méis escuros. Já o Na e o K influenciam a separação no sentido 


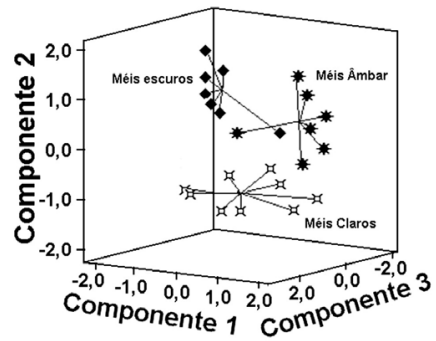

Figura 3. Resultados da análise de componentes principais mostrando o gráfico dos escores das características físico-químicas nas amostras estudadas (CP1xCP2xCP3): amostras de méis claros (Ъ), méis âmbar (*) e méis escuros (•)

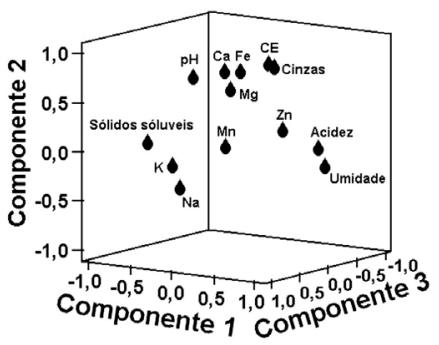

Figura 4. Comportamento das variáveis em relação aos três primeiros componentes principais

contrário, ou seja, separam as amostras de méis claros, confirmando os resultados apresentados na matriz de correlação (Tabela 2). Desta forma, a primeira componente distingue a cor dos méis em função das concentrações dos íons metálicos presentes nas amostras e a segunda componente está relacionada com os parâmetros que definem o tempo de prateleira do produto (umidade, $\mathrm{pH}$, acidez e sólidos solúveis).

\section{CONCLUSÕES}

Os méis produzidos na região sudoeste apresentam grande diversidade quanto à aparência, principalmente em relação ao aspecto cor, mas todas as cores apresentadas pelas amostras estudadas estavam em conformidade com a legislação brasileira.

Os méis com tonalidades mais escuras apresentaram maior concentração de minerais, valores de $\mathrm{pH}$ mais elevados e uma estreita relação com os elementos cálcio e ferro. Os méis com tonalidades mais claras se correlacionaram com os teores de $\mathrm{Na}$, um dos principais elementos constituintes das cinzas dos méis.

As aplicações das técnicas de análise de agrupamento hierárquica e análise de componentes principais demonstraram a relação existente entre as cores dos méis e a sua composição físico-química.

\section{MATERIAL SUPLEMENTAR}

No material suplementar, disponível gratuitamente em http:// quimicanova.sbq.org.br na forma de arquivo PDF, encontra-se o mapa da área em estudo (Figura 1S).

\section{AGRADECIMENTOS}

À Fundação de Amparo à Pesquisa do Estado da Bahia (FAPESB) pelo apoio financeiro.

\section{REFERÊNCIAS}

1. Coelho, M. de S.; Silva, J. H. V. da; Oliveira, E. R. A. de; Araújo, J. A. de; Lima, M. R. de; Caatinga 2008, 21, 1.

2. http://www.agrosoft.org.br/agropag, acessada em Março 2009.

3. Montenegro, S. B.; Avallone, C. M.; Crazov, A.; Aztarbe, M.; Resumos da Reunión Anual de Comunicaciones Científicas y Tecnológicas, Chaco, Argentina, 2005.

4. Pérez, A. O. H.; Tesis de Licenciatura, Universidad Austral de Chile, Chile, 2004.

5. Almeida, D. de.; Dissertação de Mestrado, Universidade Estadual de São Paulo, Brasil, 2002.

6. BRASIL. Ministério da Agricultura, Pecuária e Abastecimento; Instrução normativa $n^{\circ} 11$, de 20 de outubro de 2000, Diário Oficial da União, 23/10/2000.

7. Stonoga, V. I. Dissertação de Mestrado, Universidade Federal do Paraná, Brasil, 1990.

8. Crane, E.; O livro do mel; $2^{\mathrm{a}}$ ed., Nobel: São Paulo, 1987.

9. Azeredo, M. A. A.; Azeredo, L. da C.; Damasceno, J. G.; Ciênc. Tecnol. Aliment. 1999, 19, 3.

10. Moura, S. G. de; Souza, D. C.; Carneiro, J. G. M.; Resumos do Congresso Brasileiro de Apicultura, Campo Grande, Brasil, 2002.

11. Bath, P. K.; Singh, N. A.; J. Agric. Food Chem. 2005, 53, 2574.

12. Santos, J. S. dos; Santos, N. S. dos; Santos, M. L. P. dos; Santos, S. N. dos; Lacerda, J. J. de J.; J. Braz. Chem. Soc. 2008,19, 502.

13. Bianchi, E. M.; La miel, características y composición, Análisis y Aduteraciones, UNSE-CEDIA: Santiago Del Estero, 1981.

14. A.O.A.C.; Official Methods of Analysis of AOAC International, $14^{\text {th }}$ ed., Association of Official Analytical Chemists: Washington, 1984.

15. Bogdanov, S.; Lüllmann, C.; Martin, P.; Ohe, W. V.; Russmann, H.; Vorwohl, G.; Persano-Oddo, L.; Sabatini, A. G.; Marcazzan, G. L.; Piro, R.; Flamini, C.; Morlot, M.; Lhéritier, J.; Borneck, R.; Marioleas, P.; Tsigouri, A.; Kerkvliet, J.; Ortiz, A.; Ivanov, T.; D’Arcy, B.; Mossel, B.; Vit, P.; Bee World 1999, 80, 61.

16. Bogdanov, S.; Harmonised Methods of the International Honey Commission. Swiss Bee Research Centre: Bern, 2002.

17. dos Santos, M. L. P.; dos Santos, J. S.; dos Santos, J. R.; de Oliveira, L. B.; Quim. Nova 2008, 31, 1997.

18. Moreti, A. C. de C. C.; Sodré, G. da S.; Marchini, L. C.; Otsuk, I. P.; Carvalho, C. A. L. de; Ciênc. Agrotec. 2009, 33, 191.

19. Mendonça, K.; Marchini, L. C.; Souza, B. de A.; Anacleto, D. de A.; Moreti, A. C. de C. C.; Cienc. Rural 2008, 38, 1748.

20. Gonzaalez-Miret, M. L.; Terrab, A.; Hernanz, D.; FernaandezRecamales, M. A.; Heredia, F. J.; J. Agric. Food Chem. 2005, 53, 2574.

21. Souza, B. de A.; Marchini, L. C.; Oda-Souza, M.; de Carvalho, C. A. L.; Alves, R. M. de O.; Quim. Nova 2009, 32, 303. 
INFLUÊNCIA DAS CARACTERÍSTICAS FÍSICO-QUÍMICAS E COMPOSIÇÃO ELEMENTAR NAS CORES DE MÉIS PRODUZIDOS POR Apis mellifera NO SUDOESTE DA BAHIA UTILIZANDO ANÁLISE MULTIVARIADA

Julian Júnio de Jesus Lacerda*, José Soares dos Santos, Shaiala Aquino dos Santos, Gisele Brito Rodrigues e Maria Lúcia Pires dos Santos

Departamento de Ciências Naturais, Universidade Estadual do Sudoeste da Bahia, Estrada do Bem Querer, km 4, 45083-900 Vitória da Conquista - BA, Brasil

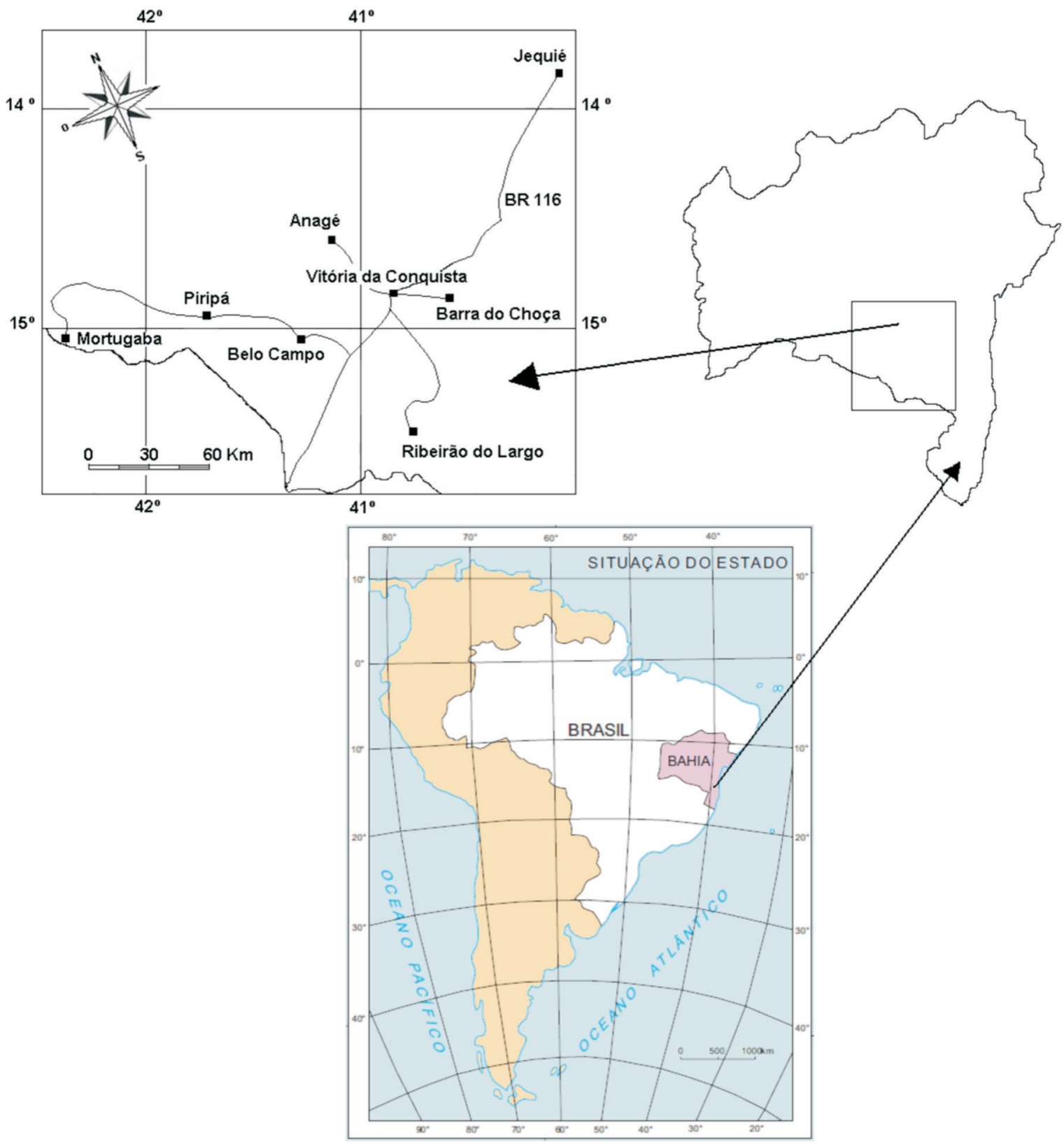

Figura 1S. Mapa dos locais de coleta das amostras de méis 